\title{
Are Points (Necessarily) Unextended?
}

\author{
Philip Ehrlich
}

Philosophy Department, Ohio University, Athens, US

Email: ehrlich@ohio.edu

(Received 04 October 2020; revised 22 April 2021; accepted 22 July 2021; first published online 12 January 2022)

\begin{abstract}
Since Euclid defined a point as "that which has no part" it has been widely assumed that points are necessarily unextended. It has also been assumed that this is equivalent to saying that points or, more properly speaking, degenerate segments, have length zero. We challenge these assumptions by providing models of Euclidean geometry where the points are extended despite the fact that the degenerate segments have null lengths, and observe that whereas the extended natures of the points are not recognizable in the given models, they can be recognized and characterized by structures that are suitable expansions of the models.
\end{abstract}

\section{Introduction}

Ever since Euclid defined a point as "that which has no part" it has been widely assumed that points are necessarily unextended (e.g., Hellman and Shapiro 2018, 189-90). It has also been assumed that, analytically speaking, this is equivalent to saying that points or, more properly speaking, degenerate segments_-segments containing a single point-have length zero (e.g., Hellman and Shapiro 2018, 189-90). ${ }^{1}$ In this paper we challenge these assumptions. We argue that neither degenerate segments having null lengths nor points satisfying the axioms of Euclidean geometry implies that Euclidean points lack extension. To make our case, we provide models of ordinary three-dimensional Euclidean geometry where the points are extended despite the fact that the corresponding degenerate segments have null lengths, as is required by the geometric axioms. The first model is used to illustrate the seemingly remarkable fact that extended points can model all of ordinary threedimensional Euclidean space, and the other two models are employed to draw attention to the fact that the internal structure of extended points may assume a variety of distinct forms. Following this, we underscore the fact that whereas the extended natures of the points are indiscernible in the just noted models and are not reflected

\footnotetext{
${ }^{1}$ Hellman and Shapiro appear to take the just noted views about points to be consequences of Euclid's characterization $(2018,189)$, rather than logical or mathematical consequences of some general concept of point.

(c) The Author(s), 2022. Published by Cambridge University Press on behalf of the Philosophy of Science Association. This is an Open Access article, distributed under the terms of the Creative Commons Attribution licence (http:// creativecommons.org/licenses/by/4.0/), which permits unrestricted re-use, distribution and reproduction, provided the original article is properly cited.
} 
by theorems of Euclidean geometry, they can be recognized as well as characterized using suitable many-sorted expansions of the given models, expansions that supplement the models with additional universes and relations defined thereon that characterize the internal structure of the points. Thus, one must draw a sharp distinction between what is true in the model of geometry, on the one hand, and what is true in the expansion (or outside) of the model, on the other. After providing illustrations of such expansions we address a pair of philosophical queries that have been raised about our models, in one case leading us to a discussion of the relative finitude and infinitude of geometrical magnitude. Finally, in an Appendix we will draw attention to a little known anticipatory model of $\mathrm{E}$. V. Huntington (1913) that we learned of long after the ideas in this paper were fully developed. To establish the consistency of his postulates for ordinary three-dimensional Euclidean geometry, Huntington introduces a "geometry of points of finite size," where, for example, "inch-spheres" serve as points $(1913,524,530)$. Like the points in our models, the extended points in Huntington's models are nondegenerate convex regions of models of paradigmatic extended geometrical spaces and are, as such, natural models of extended spatial regions, which in principle might be nondegenerate regions of physical space. Unlike the envisioned hypothesized physical counterparts of the abstract geometrical models that are our primary focus, however, Huntington's just noted models are incompatible with certain scalar aspects of Newtonian (physical) space, where the points are assumed to be infinitesimal relative to the standard inch.

\section{Euclidean geometry}

By three-dimensional elementary Euclidean geometry we mean a system of axioms that is collectively equivalent to the three-dimensional analog of Hilbert's ([1902] 1971) axioms for standard Euclidean plane geometry save his continuity axioms, the latter being the axioms that limit the models to isomorphic copies of two-dimensional Cartesian geometry over the ordered field $\mathbb{R}$ of real numbers. For our purpose, it is especially convenient to employ Tarski's system $\mathcal{P}_{3}$ (Tarski 1959, axioms $A_{1}-A_{12}$ and note 5; Schwabhäuser, Szmielew, and Tarski 1983, 10-24), which is formulated as a first-order theory (with the equality symbol "=" treated as logical identity), in which points are the sole primitive individuals and the only nonlogical predicates employed in the axioms are a ternary primitive predicate $B$ (where "Bxyz" is read $y$ lies between $x$ and $z$, the case when $y$ coincides with $x$ or $z$ not being excluded) and a quarternary primitive predicate $\equiv$ (where " $x y \equiv z u$ " is read $x$ is as distant from $y$ as $z$ is from $u$ ). The line through distinct points $a$ and $c$ in $\mathcal{P}_{3}$ is the set of all points $b$ such that $B a b c \vee B b c a \vee B c a b$, and a segment $a c$ is the set of all points $b$ such that $B a b c$, the segment being degenerate if $a=c$.

The conception that bridges the gap between the domains of number and of Euclidean magnitude is the classical notion of a Cartesian space over an ordered field. In the latest version of Tarski's framework (Schwabhäuser, Szmielew, and Tarski 1983, 16), the three-dimensional version of this familiar concept assumes the following form.

A three-dimensional Cartesian space over an ordered field $\langle F,+, \cdot,\langle, 0,1\rangle$ is a structure $C_{3}(F)=:\left\langle A_{F}, B_{F}, \equiv_{F}\right\rangle$, where the betweenness relation $B_{F}$ and the equidistance relation $\equiv_{F}$ are defined on $A_{F}=\left\{\left(x_{1}, x_{2}, x_{3}\right): x_{1}, x_{2}, x_{3} \in F\right\}$ by the stipulations: 
$B_{F} x y z$ if and only if there is $a, f \in F$ for which $0 \leq \lambda \leq 1$ and

$$
\begin{gathered}
y_{\alpha}-x_{\alpha}=\lambda\left(z_{\alpha}-x_{\alpha}\right) \text { for } \alpha=1,2,3 \\
x y \equiv{ }_{F} u v \text { if and only if } \sum_{\alpha=1}^{3}\left(x_{\alpha}-y_{\alpha}\right)^{2}=\sum_{\alpha=1}^{3}\left(u_{\alpha}-v_{\alpha}\right)^{2} .
\end{gathered}
$$

The relation between Cartesian spaces and models of $\mathcal{P}_{3}$ is given by the following well-known result that has its roots in the work of Hilbert (1899; [1902] 1971).

Representation Theorem for $\mathcal{P}_{3} . M$ is a model of $\mathcal{P}_{3}$ if and only if $M$ is isomorphic to $C_{3}(F)$, where $F$ is a Pythagorean ordered field-an ordered field $F$, in which $\sqrt{a^{2}+b^{2}} \in F$ whenever $a, b \in F$.

The central ingredient in the proof of the only if portion of the above theorem is the result: if $l$ is the line in a model $M$ of $\mathcal{P}_{3}$ containing the distinct points $o$ and $e$, then by letting the segments oo and oe serve as the zero (segment) and unit (segment), respectively, and by appealing to familiar geometric constructions, one can define operations $+_{l}$ and $\cdot_{l}$ on, and a relation $<_{l}$ between, the directed segments of $l$ (having $o$ as an end point) so that the resulting structure

$$
F_{o e}^{l}=\left\langle l,+_{l}, \cdot_{l},<_{l}, 00, o e\right\rangle,
$$

is a Pythagorean ordered field that is within isomorphism independent of the choices of $l, o$, and $e \neq 0$. Employing $F_{o e}^{l}$ together with the triple of mutually perpendicular lines $l, l^{\prime}$, and $l^{\prime \prime}$ of $M$ having the point $o$ in common, one then introduces a system of Cartesian coordinates and shows that $M$ is isomorphic to $C_{3}\left(F_{o e}^{l}\right)$. Henceforth, we will refer to $F_{o e}^{l}$ (or an isomorphic copy thereof) as the characteristic ordered field of $M .^{2}$

To obtain a categorical axiomatization of ordinary three-dimensional Euclidean geometry one need only supplement $\mathcal{P}_{3}$ with the Dedekind Continuity Axiom (Tarski $1959,18)$. Henceforth we will refer to the axiomatization thus obtained as $\mathcal{E}_{3}$. Since the characteristic ordered field of a model of $\mathcal{E}_{3}$ is isomorphic to $\mathbb{R}$, we now have the familiar

Representation Theorem for $\mathcal{E}_{3} . M$ is a model of $\mathcal{E}_{3}$ if and only if $M$ is isomorphic to $C_{3}(\mathbb{R})$.

The constructions of our models of $\mathcal{E}_{3}$ make use of a familiar model-theoretic technique that constructs models vis-à -vis isomorphisms. Since the Dedekind Continuity Axiom is a second-ordered assertion, the discussion of these models takes place in classical second-order logic.

\section{First model of $\mathcal{E}_{3}$ with extended points}

For each point $x=\left(x_{1}, x_{2}, x_{3}\right) \in A_{\mathbb{R}}=\mathbb{R}^{3}$, let $\mathbf{x}$ be a copy of $\left\langle A_{\mathbb{R}}, B_{\mathbb{R}}, \equiv_{\mathbb{R}}\right\rangle=C_{3}(\mathbb{R})$ indexed by $x$; that is, let

\footnotetext{
${ }^{2}$ In place of $F_{o e}^{l}$, Tarski $(1959,21-22)$ employs the isomorphic copy thereof that results from replacing each of the directed segments $o x$ and yo of $F_{o e}^{l}$ with their corresponding endpoints $x$ and $y$, and other authors (e.g., Hartshorne 2000, ch. 4) employ an isomorphic copy of $F_{o e}^{l}$ based on equivalence classes of congruent directed segments of the space. For the construction of $F_{o e}^{l}$ itself, see, for example, Lingenberg and Bauer (1974, 90-91).
} 


$$
\mathbf{x}:=C_{3}(\mathbb{R})_{x},
$$

which, on occasion, we will also write as $\left\langle\mathbb{R}_{x}^{3}, B_{x}, \equiv_{x}\right\rangle$. To ensure that $\mathbf{x} \cap \mathbf{y}=\emptyset$ whenever $x \neq y$, we may suppose, for example, that $\mathbb{R}_{x}^{3}:=\left\{a_{x}:=(a, x): a \in \mathbb{R}^{3}\right\}$. Further, let $\Pi=\left\{\mathbf{x}: x \in \mathbb{R}^{3}\right\}$ and define betweenness and equidistance relations $B$ and $\equiv$ on $\Pi$ by stipulating: for all $\mathbf{a}, \mathbf{b}, \mathbf{c}, \mathbf{d} \in \Pi$,

Babc if and only if $B_{\mathbb{R}} a b c$,

$$
\mathbf{a b} \equiv \mathbf{c d} \text { if and only if } a b \equiv{ }_{\mathbb{R}} c d .
$$

Since the mapping $f: \mathbb{R}^{3} \rightarrow \Pi$ defined by the condition $f(x)=\mathbf{x}=C_{3}(\mathbb{R})_{x}$ for all $x \in \mathbb{R}^{3}$ is an isomorphism from $C_{3}(\mathbb{R})$ onto $\langle\Pi, B, \equiv\rangle$, it follows from the above construction and the Representation Theorem for $\mathcal{E}_{3}$ that:

Theorem 1. $\langle\Pi, B, \equiv\rangle$ is a model of $\mathcal{E}_{3}$, each of whose points is itself a model of $\mathcal{E}_{3}$.

Moreover, since the characteristic ordered field of $\langle\Pi, B, \equiv\rangle$ is isomorphic to $\mathbb{R}$, each segment of $\langle\Pi, B, \equiv\rangle$ is assigned a nonnegative real-valued length that is zero if and only if the segment is degenerate. Accordingly, we have

Corollary 1. Despite the fact that each point in the model $\langle\Pi, B, \equiv\rangle$ of $\mathcal{E}_{3}$ is itself a model of $\mathcal{E}_{3}$, each degenerate segment of $\langle\Pi, B, \equiv\rangle$ has length zero in the model.

The reader will notice that if in the above construction and the arguments for Theorem 1 and Corollary 1 , we replace $\mathbf{x}:=C_{3}(\mathbb{R})_{x}$ with $\mathbf{x}:=\mathbb{R}_{x}^{3}$, the resulting arguments continue to hold, and we obtain the following results:

Theorem $\mathbf{1}^{\dagger} .\langle\Pi, B, \equiv\rangle$ is a model of $\mathcal{E}_{3}$, each of whose points (together with the betweenness and equidistance relations defined on it) is itself a model of $\mathcal{E}_{3}$.

Corollary $\mathbf{1}^{\dagger}$. Despite the fact that each point in the model $\langle\Pi, B, \equiv\rangle$ of $\mathcal{E}_{3}$ (together with the betweenness and equidistance relations defined on it) models $\mathcal{E}_{3}$, each degenerate segment of $\langle\Pi, B, \equiv\rangle$ has length zero in the model.

The only difference between the two approaches is that in the first the points are models of $\mathcal{E}_{3}$, and in the second they are the universes of the models. In our treatments of the subsequent two models of $\mathcal{E}_{3}$, we will limit the discussion to the first approach, with the understanding that the second approach is available in those cases as well. In section 9, we will draw attention to a consequence that results from the difference in the two approaches.

\section{Archimedean and non-Archimedean ordered fields and models of $\mathcal{E}_{3}$}

Unlike the above construction, the next construction of a model of $\mathcal{E}_{3}$ containing extended points makes use of a non-Archimedean ordered field. To prepare the way for the latter construction as well as for some material in subsequent sections we will recall some of the basics of Archimedean and non-Archimedean ordered fields and their underlying ordered additive groups. 
An ordered field $\langle F,+, \cdot,\langle, 0,1\rangle$ is said to be Archimedean if its ordered additive group $\langle F,+,<, 0\rangle$ satisfies the Archimedean condition: whenever $a$ and $b$ are nonzero members of $F$ where $|a|<|b|$, there is a positive integer $n$ such that $n|a|>|b|$. If $F$ is not Archimedean, it is said to be non-Archimedean. Since every ordered field contains a unit element, written "1," an element $a$ of $F$ may be said to be infinitesimal if $|a|<1 / n$ for every positive integer $n$, and it may be said to be infinite if $|a|>n \cdot 1$ for every positive integer $n$. An ordered field is Archimedean if and only if it contains neither infinite nor nonzero infinitesimal elements. NonArchimedean ordered fields, by contrast, contain infinite as well as nonzero infinitesimal elements. Zero is the sole infinitesimal element of an Archimedean ordered field.

If $a$ and $b$ are nonzero members of the ordered additive group of $\langle F,+, \cdot,<, 0,1\rangle$, then $a$ is said to be Archimedean equivalent to $b$ (or finite relative to $b$ ) if there are positive integers $m$ and $n$ such that $m|b|>|a|$ and $n|a|>|b|$. Archimedean equivalence partitions the elements of $F-\{0\}$ into disjoint classes called Archimedean classes. The term "Archimedean class" is intended to indicate that within a given class the Archimedean condition holds. If $a, b \in F$ are not Archimedean equivalent, then $a$ is said to be infinitesimal (in absolute value) relative to $b$ and $b$ is said to be infinite (in absolute value) relative to $a$, if $|a|<|b|$. In accordance with these conventions, 0 is infinitesimal (in absolute value) relative to every member of $F-\{0\}$. Given the absence of a unit element, the notions of infinite and nonzero infinitesimal members of $\langle F,+,<, 0\rangle$ are not well-defined. On the other hand, the infinite and infinitesimal members of $\langle F,+, \cdot,\langle, 0,1\rangle$ are the members of the field that are (in absolute value) infinite and infinitesimal, respectively, relative to the unit element.

A model of $\mathcal{P}_{3}$ may be said to be Archimedean if and only if its characteristic ordered field is Archimedean (see section 2). Therefore, since an ordered field is Archimedean if and only if it is isomorphic to a subfield of $\mathbb{R}$, every model of $\mathcal{E}_{3}$ is Archimedean. As such, the nondegenerate segments of a model of $\mathcal{E}_{3}$ are all finite relative to one another and infinite relative to the degenerate segments of the model.

\section{The non-Archimedean ordered field $\mathcal{L}$}

The construction that underlies our second model can be carried out using any nonArchimedean Pythagorean ordered field that contains an isomorphic copy of the ordered field of real numbers. The following familiar example of such a field was introduced by Tullio Levi-Civita in the first of his groundbreaking works (1892-93; 1898) that placed Giuseppe Veronese's pioneering investigation of nonArchimedean geometry (1891) on an algebraic foundation.

Let $\mathcal{L}$ be the collection of all power series

$$
\sum_{\alpha<\beta} r_{\alpha} t^{\gamma_{\alpha}}
$$

where $\left\{\gamma_{\alpha}: \alpha<\beta \leq \omega\right\}$ is a (possibly empty) strictly decreasing sequence of members of $\mathbb{R}$ that is coinitial with $\mathbb{R}$ if $\beta=\omega$, and $\left\{r_{\alpha}: \alpha<\beta\right\}$ is a sequence of members of $\mathbb{R}-\{0\} . \mathcal{L}$ is a non-Archimedean ordered field (with the empty series serving as the zero of the field) when the order is defined lexicographically and the sums and products are defined à la polynomials, with $t^{\gamma} \cdot t^{\gamma \prime}=t^{\gamma+\gamma^{\prime}}$ for all $\gamma, \gamma^{\prime} \in \mathbb{R}$. If we insert "dummy" terms with zeros for coefficients to permit a uniform representation of members of $\mathcal{L}$, then these conditions (written $+_{\mathcal{L}}, \cdot_{\mathcal{L}}$, and $<_{\mathcal{L}}$ ) may be stated more 
formally as follows, where,$+ \cdot$, and $<$ designate the standard addition, multiplication, and order in $\mathbb{R}$.

$$
\begin{gathered}
\sum_{\gamma \in \mathbb{R}} a_{\gamma} t^{\gamma}+\sum_{\mathcal{L}} \sum_{\gamma \in \mathbb{R}} b_{\gamma} t^{\gamma}=\sum_{\gamma \in \mathbb{R}}\left(a_{\gamma}+b_{\gamma}\right) t^{\gamma}, \\
\sum_{\gamma \in \mathbb{R}} a_{y} t^{\gamma} \cdot \mathcal{L} \sum_{\gamma \in \mathbb{R}} b_{y} t^{\gamma}=\sum_{\gamma \in \mathbb{R}}\left[\sum_{\substack{(\mu, \nu) \in \mathbb{R} \times \mathbb{R} \\
\mu+\nu=\gamma}} a_{\mu} b_{\nu}\right] t^{\gamma}, \\
\sum_{\gamma \in \mathbb{R}} a_{\gamma} t^{\gamma}<\sum_{\mathcal{L}} \sum_{\gamma \in \mathbb{R}} b_{\gamma} t^{\gamma}, \text { if } a_{\gamma}=b_{\gamma} \text { for all } \gamma>\text { some } \tau \in \mathbb{R} \text { and } a_{\tau}<b_{\tau} .
\end{gathered}
$$

In virtue of the lexicographical ordering, the infinitesimals of $\mathcal{L}$ are the members of $\mathcal{L}$ whose greatest exponent $\gamma_{0}$ is negative. Since 0 corresponds to the empty series, this vacuously holds for 0 .

\section{Second model of $\mathcal{E}_{3}$ with extended points}

Let $\mathcal{L}$ be defined as above, and, henceforth, by $\hat{\mathbb{R}}$ we mean the isomorphic copy of $\mathbb{R}$ in $\mathcal{L}$ consisting of $\{0\} \cup\left\{x \in \mathcal{L}: x=r t^{0}\right.$ for somer $\left.\in \mathbb{R}-\{0\}\right\}$ with the addition, multiplication, and order inherited from $\mathcal{L}$. First note that, since $\mathcal{L}$ is a Pythagorean ordered field (e.g., Robinson 1973, 92), $\mathcal{C}_{3}(\mathcal{L})$ is a model of $\mathcal{P}_{3}$. Moreover, since $\hat{\mathbb{R}} \subset \mathcal{L}$ and $\hat{\mathbb{R}}$ is isomorphic to $\mathbb{R}, C_{3}(\hat{\mathbb{R}}) \subset C_{3}(\mathcal{L})$ and $C_{3}(\hat{\mathbb{R}})$ is isomorphic to $C_{3}(\mathbb{R})$.

Let $\mathcal{L}_{I}$ be the set of infinitesimals of $\mathcal{L}$, and for each point $x=\left(x_{1}, x_{2}, x_{3}\right) \in \mathbb{R}^{3}$, let

$$
\mathbf{x}:=\left\{\left(x_{1}+\delta_{1}, x_{2}+\delta_{2}, x_{3}+\delta_{3}\right) \in \mathcal{L}^{3}: \delta_{1}, \delta_{2}, \delta_{3} \in \mathcal{L}_{I}\right\},
$$

together with the restrictions thereto of the betweenness and equidistance relations $B_{\mathcal{L}}$ and $\equiv_{\mathcal{L}}$ from $C_{3}(\mathcal{L})$. Thus, for each point $x \in \hat{\mathbb{R}}^{3}$, the universe of $\mathbf{x}$ is the infinite set of all points in $C_{3}(\mathcal{L})$ that are an infinitesimal distance from $x$, including $x$ itself. Using this, one may readily show: $\mathbf{x} \cap \mathbf{y}=\emptyset$ for distinct $x, y \in \hat{\mathbb{R}}^{3}$; and for each $x \in \hat{\mathbb{R}}^{3}, \mathbf{x}$ is a convex region of $C_{3}(\mathcal{L})$ in the sense that for all $a, b, c \in C_{3}(\mathcal{L}), b \in \mathbf{x}$ whenever $a, c \in \mathbf{x}$ and $B_{\mathcal{L}} a b c$. Moreover, $\mathbf{x}$ is three-dimensional, as is evident from its containment of the three mutually orthogonal (nondegenerate) segments of $C_{3}(\mathcal{L})$ having endpoints

$$
\begin{aligned}
& \left(x_{1}, x_{2}, x_{3}\right),\left(x_{1}+\delta, x_{2}, x_{3}\right) \\
& \left(x_{1}, x_{2}, x_{3}\right),\left(x_{1}, x_{2}+\delta, x_{3}\right) \\
& \left(x_{1}, x_{2}, x_{3}\right),\left(x_{1}, x_{2}, x_{3}+\delta\right),
\end{aligned}
$$

where $\delta$ is a nonzero member of $\mathcal{L}_{I}$.

Now let

$$
\hat{\Pi}:=\left\{\mathbf{x}: x \in \hat{\mathbb{R}}^{3}\right\}
$$

and define betweenness and equidistance relations $\hat{B}$ and $\hat{\equiv}$ on $\hat{\Pi}$ by the conditions: for all $\mathbf{a}, \mathbf{b}, \mathbf{c}, \mathbf{d} \in \hat{\Pi}$,

$\hat{B} \mathbf{a b c}$ if and only if $B_{\mathcal{L}} a b c$, 


$$
\mathbf{a b} \triangleq \mathbf{\equiv d} \text { if and only if } a b \equiv{ }_{\mathcal{L}} c d .
$$

Also let $f: \hat{\mathbb{R}}^{3} \rightarrow \hat{\Pi}$ be the mapping defined by the condition: $f(x)=\mathbf{x}$ for all $x \in \hat{\mathbb{R}}^{3}$. Plainly, $f$ is an isomorphism from $C_{3}(\hat{\mathbb{R}})$ onto $\langle\hat{\Pi}, \hat{B}, \hat{\equiv}\rangle$; and so, since $C_{3}(\hat{\mathbb{R}})$ is isomorphic to $C_{3}(\mathbb{R})$, it follows that $\langle\hat{\Pi}, \hat{B}, \hat{\equiv}\rangle$ is isomorphic to $C_{3}(\mathbb{R})$. But then, in virtue of the Representation Theorem for $\mathcal{E}_{3}$ and the just described construction, we have

Theorem 2. $\langle\hat{\Pi}, \hat{B}, \hat{\equiv}\rangle$ is a model of $\mathcal{E}_{3}$, each of whose points is a convex three-dimensional region of $C_{3}(\mathcal{L})$.

Moreover, by invoking the argument employed in section 3 to establish Corollary 1 , we have

Corollary 2. Despite the fact that each point in the model $\langle\hat{\Pi}, \hat{B}, \hat{\equiv}\rangle$ of $\mathcal{E}_{3}$ is a convex threedimensional region of $C_{3}(\mathcal{L})$, each degenerate segment of $\langle\hat{\Pi}, \hat{B}, \hat{\equiv}\rangle$ has length zero in the model.

\section{Hjelmslev's nilpotent infinitesimalist continuum}

As in the second construction of a model of $\mathcal{E}_{3}$ with extended points, in our third construction the points are convex three-dimensional regions of an overarching geometric space. Here the overarching space is an affine Hjelmslev space over the lexicographically (totally) ordered ring $\mathbb{R}[\varepsilon]$ of dual numbers, the three-dimensional counterpart of the nilpotent infinitesimalist geometric continuum introduced by Johannes Hjelmslev (1923). To prepare the way for the construction, we begin with a brief overview of the system of dual numbers and the three-dimensonal affine Hjelmslev space defined on it. ${ }^{3}$

The system $\mathbb{R}[\varepsilon]$ of dual numbers, which was introduced by William Clifford (1873), is a commutative ring with identity consisting of

$$
\left\{r_{0}+r_{1} \varepsilon: r_{0}, r_{1} \in \mathbb{R}\right\}
$$

with sums and products defined in the manner of polynomials, with $\varepsilon \neq 0$ and $\varepsilon^{2}=0$. Thus, for any two dual numbers $a_{0}+a_{1} \varepsilon$ and $b_{0}+b_{1} \varepsilon$

$$
\begin{gathered}
\left(a_{0}+a_{1} \varepsilon\right)+\left(b_{0}+b_{1} \varepsilon\right)=\left(a_{0}+b_{0}\right)+\left(a_{1}+b_{1}\right) \varepsilon \\
\left(a_{0}+a_{1} \varepsilon\right)\left(b_{0}+b_{1} \varepsilon\right)=a_{0} b_{0}+\left(a_{0} b_{1}+a_{1} b_{0}\right) \varepsilon .
\end{gathered}
$$

The element $\varepsilon$ in $\mathbb{R}[\varepsilon]$ is a nilpotent, an element $x$ such that $x^{n}=0$ for some positive integer $n$. The least $n$ for which $x^{n}=0$ is the index of nilpotency of $x$. The index of nilpotency of $\varepsilon$ in $\mathbb{R}[\varepsilon]$ is 2 . Nonzero nilpotent elements of index $n$ have many applications in algebra, one of them being a convenient way of representing quantities up to infinitesimal order $n$. When nilpotents are thus interpreted, they are referred to as

\footnotetext{
${ }^{3}$ Like Hjelmslev geometries, Synthetic Differential Geometry (e.g., Kock 1981) makes use of nilpotent infinitesimals. However, unlike the latter theory, whose underlying logic is intuitionistc to avoid outright inconsistency with classical logic, the underlying logic of Hjelmslev geometries is classical. For further discussion of these matters, see Ehrlich (2021).
} 
nilpotent infinitesimals. The number systems we have hitherto considered have no nonzero nilpotent elements.

Inspired by the view of the pre-Socratic philosopher Protagoras, as recounted in Aristotle's Metaphysics (III: 2, 998a), Hjelmslev held that the axiom that two straight lines always share at most one point is incompatible with perceptual experience, as is the assertion that a circle and a line tangent to it meet at a single point (Hjelmslev 1923, 1-2). This led him to devise a "geometry of reality" (Hjelmslev 1916) or a "natural geometry" (Hjelmslev 1923), as he later called it, whose subject is the lines and circles of perception constructed with real rulers and compasses. He further devised (Hjelmslev 1923, 12-13) the above-mentioned abstraction of the latter, coordinated by $\mathbb{R}[\varepsilon]$, that is a prototype of what are today called affine Hjelmslev geometries. In these geometries, which are coordinated by affine Hjelmslev rings, while each pair of distinct points has a line joining them, it need not be unique. Indeed, a pair of distinct points may lie on a pair of distinct lines; when this happens the points are said to be neighboring points, and the lines are said to be neighboring lines, the two notions of neighbor being equivalence relations. Remote (or nonneighboring) points, by contrast, are joined by a unique line, and remote (or nonneighboring) lines that intersect, intersect in a unique point. In these geometries a circle and a line tangent to it intersect in a nondegenerate "infinitesimal segment" of neighboring points.

Formally speaking, in their standard formulations, affine Hjelmslev planes are structures $\langle\mathbb{P}, \mathbb{L}, \|, I\rangle$ (satisfying a set of axioms-see references below) where $\mathbb{P}$ is a set of points, $\mathbb{L}$ is a set of subsets of $\mathbb{P}$ called lines, $\|$ is a parallel relation defined on $\mathbb{L}$, and $I$ is an incidence relation defined on pairs $(a, b) \in \mathbb{P} \times \mathbb{L}$. Moreover, and of central importance, the axioms ensure that the geometrical relational structure $\left\langle\mathbb{P}^{*}, \mathbb{L}^{*}, \|^{*}, I^{*}\right\rangle$, with the induced relations $\|^{*}$ and $I^{*}$, obtained from $\langle\mathbb{P}, \mathbb{L}, \|, I\rangle$ by replacing each $p \in \mathbb{P}$ and each $l \in \mathbb{L}$ with the equivalence classes consisting of all neighboring points of $p$ and all neighboring lines of $l$, respectively, is isomorphic to an ordinary affine geometric counterpart, in which two points determine a line and lines that intersect do so in a unique point. In the case of Hjelmslev's aforementioned prototype, which is coordinated by $\mathbb{R}[\varepsilon]$, the affine counterpart is (as Hjelmslev observed) isomorphic to the standard affine Euclidean plane over $\mathbb{R}$. It is the existence of this isomorphism that motivates our third construction.

Hjelmslev's plane over $\mathbb{R}[\varepsilon]$, henceforth $\mathrm{H}_{2}(\mathbb{R}[\varepsilon])$, consists of all ordered pairs $(A, B) \in \mathbb{R}[\varepsilon] \times \mathbb{R}[\varepsilon]$. As usual, a straight line of $H_{2}(\mathbb{R}[\varepsilon])$ is defined by a first-degree equation

$$
A x+B y+C=0,
$$

where now, however, $A, B, C \in \mathbb{R}[\varepsilon]$, it being understood that at least one of $A, B$ is not in the ideal $\mathbb{R}[\varepsilon]_{I}=\{0+r \varepsilon: r \in \mathbb{R}\}$ of nilpotent infinitesimal elements of $\mathbb{R}[\varepsilon]$. In $H_{2}(\mathbb{R}[\varepsilon])$, a pair of points $\left(a_{1}, a_{2}\right)$ and $\left(b_{1}, b_{2}\right)$ are neighbors if and only if $b_{1}-a_{1}$ and $b_{2}-a_{2}$ are elements of $\mathbb{R}[\varepsilon]_{I}$.

$\mathbb{R}[\varepsilon]$ admits a relational expansion to a non-Archimedean totally ordered ring, where the order $<$ is defined lexicographically by the condition: $a_{0}+a_{1} \varepsilon<b_{0}+b_{1} \varepsilon$ if $a_{0}<_{\mathbb{R}} b_{0}$ or $a_{0}=b_{0}$ and $a_{1}<{ }_{\mathbb{R}} b_{1}$. In virtue of the just noted ordering, the points on a line of $H_{2}(\mathbb{R}[\varepsilon])$ are themselves totally ordered and admit a natural betweenness relation $B_{\mathbb{R}[\varepsilon]}$ defined by the condition: for all points $x, y, z$ 
on a line $l$ of $H_{2}(\mathbb{R}[\varepsilon])$ totally ordered by $<$ l, $y$ lies between $x$ and $z$ if either $x<y<{ }_{l} z$ or $z<y<x_{l} x^{4}$

Examples of neighboring lines in $H_{2}(\mathbb{R}[\varepsilon])$ are

$$
y=0 \text { and } y=\varepsilon x
$$

which intersect at all neighboring points $(r \varepsilon, 0)$ where $r \in \mathbb{R}$. And similarly, the circle defined by the equation

$$
A x^{2}+B y^{2}=1
$$

intersects the line defined by the equation

$$
y=1
$$

at all neighboring points $(r \varepsilon, 1)$ where $r \in \mathbb{R}$. In each of these cases, the set of neighboring points constituting the segment of intersection is isomorphic to $\mathbb{R}$ considered as an ordered set (Hjelmslev 1923, 13).

Inspired by these and related ideas of Hjelmslev $(1923,1929)$, the axiomatic theory of Hjelmslev planes (and corresponding theory of Hjelmslev rings) was developed by Klingenberg $(1954,1954 a, 1955)$ and was extended to higher dimensional spaces by Kreuzer $(1987,1988)$. Our third construction, to which we now turn, makes use of the three-dimensional analog of $H_{2}(\mathbb{R}[\varepsilon])$, henceforth denoted $H_{3}(\mathbb{R}[\varepsilon])$, where the basic ideas and concepts of $H_{2}(\mathbb{R}[\varepsilon])$ readily extend to the three-dimensional case. In particular, in $H_{3}(\mathbb{R}[\varepsilon])$, a pair of points $\left(a_{1}, a_{2}, a_{3}\right)$ and $\left(b_{1}, b_{2}, b_{3}\right)$ are neighbors if and only if $b_{1}-a_{1}, b_{2}-a_{2}$, and $b_{3}-a_{3}$ are elements of $\mathbb{R}[\varepsilon]_{I}$, and given a point and a line in a plane of $H_{3}(\mathbb{R}[\varepsilon])$ there is one line perpendicular to the line through the point (Kreuzer 1987, 1988).

\section{Third model of $\mathcal{E}_{3}$ with extended points}

Let $\tilde{\mathbb{R}}$ be the isomorphic copy of $\mathbb{R}$ in $\mathbb{R}[\varepsilon]$ consisting of $\{r+0 \varepsilon: r \in \mathbb{R}\}$ with the addition, multiplication, and order inherited from $\mathbb{R}[\varepsilon]$, and for each $x=\left(x_{1}, x_{2}, x_{3}\right) \in \tilde{\mathbb{R}}^{3}$, let

$$
\mathbf{x}:=\left\{\left(x_{1}+\delta_{1}, x_{2}+\delta_{2}, x_{3}+\delta_{3}\right) \in \mathbb{R}[\varepsilon]^{3}: \delta_{1}, \delta_{2}, \delta_{3} \in \mathbb{R}[\varepsilon]_{I}\right\}
$$

together with the restriction thereto of the aforementioned betweennesss relation $B_{\mathbb{R}[\varepsilon]}$ defined on $H_{3}(\mathbb{R}[\varepsilon])$. Finally, let

$$
\tilde{\Pi}:=\left\{\mathbf{x}: x \in \tilde{\mathbb{R}}^{3}\right\} .
$$

For each $x \in \tilde{\mathbb{R}}^{3}, \mathbf{x}$ is the (infinite) set of all points in $H_{3}(\mathbb{R}[\varepsilon])$ that are neighbors of $x$, including $x$ itself. Since the neighbor relation on points is an equivalence relation that partitions $\mathbb{R}[\varepsilon]^{3}$ into disjoint classes, it follows that for distinct $x, y \in \tilde{\mathbb{R}}^{3}, \mathbf{x} \cap \mathbf{y}=\emptyset$ and

\footnotetext{
${ }^{4}$ For details, see Baker, Lane, Lorimer, and Laxton $(1984,25)$. Without loss of generality, a nonstrict betweenness relation (à la Tarski) may also be defined on $\mathbb{R}[\varepsilon]$ by replacing $<_{l}$ with $\leq_{l}$ in the above definition. However, since we will later appeal to a result from the literature on affine Hjelmslev geometries that is formulated in terms of the strict betweenness relation, we have adopted the latter relation.
} 


$$
\bigcup_{\mathbf{x} \in \tilde{\Pi}} \mathbf{x}=\mathbb{R}[\varepsilon]^{3}
$$

Moreover, by a result of Baker, Lane, Lorimer and Laxton $(1984,20-22)^{5}$, for each $x \in \tilde{\mathbb{R}}^{3}, \mathbf{x}$ is a convex subclass of $H_{3}(\mathbb{R}[\varepsilon])$ in the sense defined in section 6 . Furthermore, $\mathbf{x}$ is three-dimensional, in virtue of its containment of triples of mutually orthogonal nondegenerate segments of $H_{3}(\mathbb{R}[\varepsilon])$, such as:

$$
\begin{gathered}
\left\{\left(x_{1}+\delta, x_{2}, x_{3}\right) \in \mathbb{R}[\varepsilon]^{3}: 0 \leq \delta \leq \varepsilon\right\} \\
\left\{\left(x_{1}, x_{2}+\delta, x_{3}\right) \in \mathbb{R}[\varepsilon]^{3}: 0 \leq \delta \leq \varepsilon\right\} \\
\left\{\left(x_{1}, x_{2}, x_{3}+\delta\right) \in \mathbb{R}[\varepsilon]^{3}: 0 \leq \delta \leq \varepsilon\right\} .
\end{gathered}
$$

By now mimicking the constructions employed in sections 3 and 6 , we define betweenness and equidistance relations $\tilde{B}$ and $\cong$ on $\tilde{\Pi}$ by the conditions: for all $\mathbf{a}, \mathbf{b}, \mathbf{c}, \mathbf{d} \in \tilde{\Pi}$,

$\tilde{B} \mathbf{a b c}$ if and only if $B_{\mathbb{R}} a b c$,

$$
\mathbf{a b} \cong \mathbf{c d} \text { if and only if } a b \equiv{ }_{\mathbb{R}} c d ;
$$

and by essentially repeating the arguments employed in the just noted sections we obtain the following analogs for $\langle\tilde{\Pi}, \tilde{B}, \cong\rangle$ of the results for $\langle\Pi, B, \equiv\rangle$ and $\langle\hat{\Pi}, \hat{B}, \hat{\equiv}\rangle$.

Theorem 3. $\langle\tilde{\Pi}, \tilde{B}, \cong\rangle$ is a model of $\mathcal{E}_{3}$, each of whose points is a convex three-dimensional region of $\mathrm{H}_{3}(\mathbb{R}[\varepsilon])$.

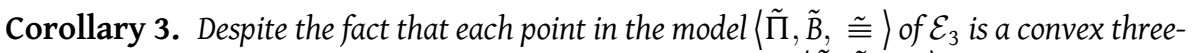
dimensional region of $H_{3}(\mathbb{R}[\varepsilon])$, each degenerate segment of $\langle\tilde{\Pi}, \tilde{B}, \cong ्$, has length zero in the model.

\section{Mathematico-philosophical reflections}

Whereas the notions of length, area, and volume measure were introduced to quantify our preanalytic notions of one-dimensional, two-dimensional, and three-dimensional spatial extension, the relation between the standard geometrical notions and the preanalytic, metageometric/metaphysical notions are not quite what is often assumed. Indeed, what our models illustrate is that it is merely the infinitesimality of degenerate segments relative to their nondegenerate counterparts (see section 4), rather than the absence of extension of points, that is implied both by the axioms of ordinary Euclidean geometry and these segments' null lengths. ${ }^{6}$ Thus, from a contemporary

\footnotetext{
${ }^{5} \mathrm{H}_{2}(\mathbb{R}[\varepsilon])$ together with the betweenness relation $B_{\mathbb{R}[\varepsilon]}$ defined thereon is an example of a preordered affine Hjelmslev plane, and Baker, Lane, Lorimer and Laxton (1984) have shown that every neighbor class of such a structure is convex in the sense defined in section 6. Their proof readily extends to the three-dimensional case.

${ }^{6}$ Beginning with Paul du Bois-Reymond $(1882,66)$, there has been a string of authors who have argued that the now standard conception of a continuum as a point-set is incoherent because it is impossible for an extended segment to be composed of unextended points. Ehrlich (2014), without appealing to the possibility of extended points, draws attention to the misguided nature of such arguments. Still, it is interesting to note that the very assumption these authors take to lead to the incoherence of the standard view is not necessitated by the standard view at all.
} 
standpoint, contrary to Euclid's intuition, a point of Euclidean geometry may very well have parts, indeed even proper parts. Moreover, as the above remarks suggest, the number zero functions quite differently as a cardinal number than as a measure number in the system of reals. So, for example, whereas a set containing zero members has no member at all, an event having probability zero-such as a randomly thrown point-tipped dart landing on a specified point of the unit interval of $\mathbb{R}$ may very well transpire, and perhaps more surprisingly still, a degenerate segment having length zero may contain a point having an internal structure that models all of Euclidean space. ${ }^{7}$

On the other hand, as we mentioned above, whereas the axioms of $\mathcal{E}_{3}$ make assertions about points, they are entirely reticent about what internal structure, if any, the points themselves have. Indeed, this is simply not the province of Euclidean geometry, at least not in its contemporary guise. Any additional assumptions about the nature of the points other than those derivable from the axioms for $\mathcal{E}_{3}$ are by their very nature extra-Euclidean. However, as we also previously mentioned, this is not to suggest that it would be impossible to suitably expand models of $\mathcal{E}_{3}$ that would have provable consequences about any internal structure the points might have. In the case of the models of $\mathcal{E}_{3}$ discussed above this can be carried out in a variety of fashions, including the following two in which the models are expanded to suitable manysorted structures with supplemental universes and relations defined thereon. Since the constructions are similar in all three cases, we will simply illustrate them with $\langle\Pi, B, \equiv\rangle$.

In the first approach, which is also the approach that more literally captures the ideas expressed in Theorem 1 and Theorem $1^{\dagger}$, one expands the Euclidean structure $\langle\Pi, B, \equiv\rangle$ to the extra-Euclidean structure

$$
\left\langle\Pi, B, \equiv,\left(\left\langle\mathbb{R}_{x}^{3}, B_{x}, \equiv_{x}\right\rangle\right)_{x \in \mathbb{R}^{3}}\right\rangle,
$$

and in the second approach, one expands $\langle\Pi, B, \equiv\rangle$ to the extra-Euclidean structure

$$
\left\langle\Pi, B, \equiv, \Pi^{*}, B^{*}, \equiv^{*}\right\rangle
$$

where

$$
\Pi^{*}=\bigcup_{x \in \mathbb{R}^{3}} \mathbb{R}_{x}^{3}, B^{*}=\bigcup_{x \in \mathbb{R}^{3}} B_{x}, \text { and } \equiv *=\bigcup_{x \in \mathbb{R}^{3}} \equiv_{x}
$$

Thus, whereas in the first approach one adds for each point $\mathbf{x} \in \Pi$ a distinct universe $\mathbb{R}_{x}^{3}$ and distinct betweenness and equidistance relations $B_{x}$ and $\equiv_{x}$ defined thereon, in the second approach one adds a single composite universe together with the corresponding composite betweenness and equidistance relations defined on it. While the structures emerging from the two approaches are formally distinct, the difference is

\footnotetext{
${ }^{7}$ Already by 1911, Federigo Enriques $(1911,101-3)$ pointed out the possibility of assigning nonzero infinitesimal probabilities to a randomly thrown point-tipped dart landing on a specified point of the unit interval $\mathbb{R}$ and thereby distinguishing measure theoretically between impossible events having probability zero, on the one hand, and certain possible though infinitely unlikely events, on the other. This idea was later taken up (without reference to Enriques) by Bernstein and Wattenberg (1969) in the context of nonstandard analysis and again by Benci, Horsten, and Wenmackers $(2013,2018)$ using their nonArchimedean probability theory. However, since the null lengths of degenerate segments are a consequence of $\mathcal{E}_{3}$, an analogous treatment of assigning nonzero measures to degenerate segments containing extended points is not available unless one ignores the Euclidean nature of the model.
} 
largely a difference in methods of bookkeeping, since each individually adjoined structure in the first approach constitutes a recognizable convex substructure of $\left\langle\Pi^{*}, B^{*}, \equiv{ }^{*}\right\rangle$ in the second. Moreover, whether one interprets $\Pi$ as a set of models of $\mathcal{E}_{3}$, as in Theorem 1, or as the set of the universes of the just noted models, as in Theorem $1^{\dagger}$, the above two expansions of $\langle\Pi, B, \equiv\rangle$ are alternative ways of formalizing the extra-Euclidean structures implicit in each of those theorems.

If we adopt the first and more literal approach, then Theorem 1 and Theorem $1^{\dagger}$ assume the following explicit forms:

Theorem 1. The structure $\left\langle\Pi, B, \equiv,\left(\left\langle\mathbb{R}_{\chi}^{3}, B_{x}, \equiv_{x}\right\rangle\right)_{x \in \mathbb{R}^{3}}\right\rangle$ is an expansion of the model $\langle\Pi, B, \equiv\rangle$ of $\mathcal{E}_{3}$, each of whose points $\left\langle\mathbb{R}_{x}^{3}, B_{x}, \equiv_{\chi}\right\rangle$ is itself a model of $\mathcal{E}_{3}$.

Theorem 1†. The structure $\left\langle\Pi, B, \equiv,\left(\left\langle\mathbb{R}_{x}^{3}, B_{x}, \equiv_{\chi}\right\rangle\right)_{x \in \mathbb{R}^{3}}\right\rangle$ is an expansion of the model $\langle\Pi, B, \equiv\rangle$ of $\mathcal{E}_{3}$, each of whose points $\mathbb{R}_{x}^{3}$ together with the betweenness and equidistance relations $B_{x}$ and $\equiv_{x}$ defined on it is itself a model of $\mathcal{E}_{3}$.

The above-described many-sorted expansions of $\langle\Pi, B, \equiv\rangle$ shed light on the internal structure of the points in $\Pi$ while leaving the model and, in particular, its points in place. Loosely speaking, whereas $\langle\Pi, B, \equiv\rangle$ provides the macroperspective of the geometric framework, $\left\langle\Pi, B, \equiv,\left(\left\langle\mathbb{R}_{x}^{3}, B_{x}, \equiv_{x}\right\rangle\right)_{x \in \mathbb{R}^{3}}\right\rangle$ and $\left\langle\Pi, B, \equiv, \Pi^{*}, B^{*}, \equiv{ }^{*}\right\rangle$ provide macro/microperspectives. There is also a complementary one-sorted structure that sheds further light on the microperspective without expanding the model itself. Since the basic construction is applicable to all three of our examples, again we will only illustrate it for the case of $\langle\Pi, B, \equiv\rangle$.

Instead of expanding the structure $\langle\Pi, B, \equiv\rangle$ with the universe $\Pi^{*}$ and the relations $B^{*}$ and $\equiv{ }^{*}$ defined thereon, one begins with the structure $\left\langle\Pi^{*}, B^{*}, \equiv{ }^{*}\right\rangle$ and expands it to the structure $\left\langle\Pi^{*}, B^{\prime}, \equiv{ }^{\prime}, B^{*}, \equiv{ }^{*}\right\rangle$, where $B^{\prime}$ and $\equiv^{\prime}$ are defined by the stipulations:

$$
\begin{gathered}
B^{\prime} a_{x} b_{y} c_{z} \text { if and only if } B_{\mathbb{R}} x y z, \\
a_{w} b_{x} \equiv{ }^{\prime} c_{y} d_{z} \text { if and only if } w x \equiv{ }_{\mathbb{R}} y z,
\end{gathered}
$$

where $w, x, y, z$ range over $\mathbb{R}^{3}$ and $a_{w}, b_{x}, c_{y}$, and $d_{z}$ range over $\mathbb{R}_{w}^{3}, \mathbb{R}_{x}^{3}, \mathbb{R}_{y}^{3}$, and $\mathbb{R}_{z}^{3}$, respectively. Unlike the tuples that comprise $B^{*}$ and $\equiv^{*}$, those that comprise $B^{\prime}$ and $\equiv$ ' do not solely relate members of individual $\mathbb{R}_{x}^{3}$ 's. Moreover, as is evident from the construction, there is an epimorphism (i.e., a surjective homomorphism) $f$ from $\left\langle\Pi^{*}, B^{\prime}, \equiv{ }^{\prime}\right\rangle$ onto $\langle\Pi, B, \equiv\rangle$, and, hence, $\langle\Pi, B, \equiv\rangle$ is isomorphic to the quotient structure $\left\langle\Pi^{*} / R, B_{R}^{\prime}, \equiv_{R}^{\prime}\right\rangle$ defined by the congruence relation $R$ generated by $f$; that is, the structure $\left\langle\Pi^{*} / R, B_{R}^{\prime},{ }^{\prime}{ }_{R}\right\rangle$ where $\Pi^{*} / R$ is the set of all equivalence classes $[x]=\left\{y \in \Pi^{*}: f(y)=f(x)\right\}, B_{R}^{\prime}$ is the set of all triples $([x],[y],[z])$ for which $(x, y, z) \in B^{\prime}$, and $\equiv_{R}^{\prime}$ is the set of all quadruples $([w],[x],[y],[z])$ for which $(w, x, y, z) \in \equiv \prime$ (e.g., Monk 1976, 385-86). Since $f$ is not $1-1$, unlike $\langle\Pi, B, \equiv\rangle$, $\left\langle\Pi^{*}, B^{\prime}, \equiv{ }^{\prime}\right\rangle$ is not a model of $\mathcal{E}_{3}$. On the other hand, $\left\langle\Pi^{*}, B^{\prime}, \equiv{ }^{\prime}\right\rangle$ may be regarded as a pre-Euclidean geometry in the sense that the quotient structure $\left\langle\Pi^{*} / R, B_{R}^{\prime},{ }_{R}{ }_{R}\right\rangle$ is a model of $\mathcal{E}_{3}$. Analogously, the members of $\Pi^{*}$ may be regarded as micropoints, though they are not points in $\langle\Pi, B, \equiv\rangle$. Moreover, the members of $\Pi^{*} / R$ are the universes of the models of $\mathcal{E}_{3}$ contained in $\Pi$, if $\Pi$ is understood as in Theorem 1, and they coincide 
with the members of $\Pi$, if $\Pi$ is understood as in Theorem $1^{\dagger}$. Accordingly, if $\langle\Pi, B, \equiv\rangle$ is understood as in Theorem $1^{\dagger}$, then $\left\langle\Pi^{*} / R, B_{R}^{\prime}, \equiv_{R}{ }_{R}\right\rangle$ is not merely isomorphic to $\langle\Pi, B, \equiv\rangle$, it is identical to $\langle\Pi, B, \equiv\rangle$.

In his mathematico-philosophical correspondence with Frege on the nature of the Euclidean axioms, Hilbert argued that the axioms constitute "only a scaffolding or schema of concepts together with their necessary relations to one another, and that the basic elements [such as points] can be thought of in any way one likes ... provided only that the requisite axioms are satisfied" (December 12, [1899] 1980, 40-41). To illustrate this he notes: "If in speaking of my points I think of some system of things, e.g. the system: love, law, chimney-sweep ... and then assume all my axioms as relations between these things, then my propositions, e.g. Pythagoras' theorem, are also valid for these things" (December 29, 1899, 40). This view, which was championed by Hilbert and a number of other late 19th- and early 20th-century geometers, has emerged as the standard construal of these matters of our day. Be that as it may, following longstanding tradition, points are still typically envisioned as unextended locations, or as locations having "no length, breadth or thickness" as Veblen ([1911] $1955,4)$ expressed it. The purpose of this paper has been to demonstrate that, longstanding tradition aside, the purported unextended nature of points is not implied by the axioms of $\mathcal{E}_{3}$. Models of $\mathcal{E}_{3}$ whose universes consist of extended points do indeed exist, though one has to go outside the Euclidean model to recognize and characterize their extended nature.

On occasion it has been suggested to the author that the constructions of models of $\mathcal{E}_{3}$ with extended points presented above are simply the workings out of the details of instances of Hilbert's quip about love, law, and chimney-sweeps ${ }^{8}$ or his less well developed quip of 1882 where he maintains: "one must be able to say 'tables, chairs, beer-mugs' each time in place of 'points, lines, planes' "(Blumenthal 1935, 402-3). While this was certainly not our intention, there is an inkling of truth in this contention, but only an inkling. As we noted in the Introduction, and as our models make clear, the extended points in our models are nondegenerate convex regions of models of paradigmatic extended geometrical spaces and are, as such, natural models of extended spatial regions - spatial regions which in principle might be nondegenerate regions of physical space. The same cannot be said of tables, love, or the like. As such, unlike the hypothesized playful models in Hilbert's quips, our models go well beyond simply pointing out that points in a model of $\mathcal{E}_{3}$ may have unintended interpretations; they challenge the deep-seated and longstanding geometric shibboleth that points are necessarily unextended and as such are necessarily devoid of internal structure.

Nevertheless, could it be that our models of $\mathcal{E}_{3}$ with extended points, particularly the first where the extended points are themselves models of $\mathcal{E}_{3}$, may exist only in a formally stipulated sense; that, for example, we lack any geometric or spatial conception of what constitutes the "betweenness" and "equidistance" between such extended points? ${ }^{9}$ We believe the answer is no, though for the sake of space we will only touch on the matter here, leaving a more detailed historical and mathematicophilosophical response for another occasion.

\footnotetext{
${ }^{8}$ This observation was first made by Patricia Blanchette.

${ }^{9}$ This query was posed by Geoffrey Hellman.
} 
To help motivate our negative response it will be useful to first consider a nonArchimedean model $M$ of $\mathcal{P}_{3}$ having characteristic ordered field $F_{o e}^{l}$ containing an isomorphic copy $\mathbb{R}_{o e}$ of $\mathbb{R}$, oo and oe being the zero and unit segments of $F_{o e}^{l}$ and hence $\mathbb{R}_{o e}$ (see sections 2, 3, and 5). Then $M$ is isomorphic to $C_{3}\left(F_{o e}^{l}\right)$, the latter of which contains a subspace $C_{3}\left(\mathbb{R}_{o e}\right)$ that is isomorphic to $C_{3}(\mathbb{R})$. Moreover, since $M$ is non-Archimedean, there are nondegenerate segments $o e^{\prime}$ and $o e^{\prime \prime}$ of $l$ such that $o e^{\prime}$ is infinitesimal relative to $o e$ and $o e^{\prime \prime}$ is infinite relative to oe. Furthermore, insofar as the characteristic ordered field of a model of $\mathcal{P}_{3}$ is (to within isomorphism) independent of the choice of unit segment, for each such pair o $e^{\prime}$ and $o e^{\prime \prime}$ there are isomorphic copies $C_{3}\left(F_{o e^{\prime}}^{l}\right)$ and $C_{3}\left(F_{o e^{\prime \prime}}^{l}\right)$ of $M$, whose characteristic ordered fields $F_{o e^{\prime}}^{l}$ and $F_{o e^{\prime \prime}}^{l}$, respectively, contain isomorphic copies $\mathbb{R}_{o e^{\prime}}$ and $\mathbb{R}_{o e^{\prime \prime}}$ of $\mathbb{R}$. Analogously, $C_{3}\left(F_{o e^{\prime}}^{l}\right)$ and $C_{3}\left(F_{o e^{\prime \prime}}^{l}\right)$, respectively, contain subspaces $C_{3}\left(\mathbb{R}_{o e^{\prime}}\right)$ and $C_{3}\left(\mathbb{R}_{o e^{\prime \prime}}\right)$, which are isomorphic to $C_{3}(\mathbb{R})$. Thus, despite the fact that the nondegenerate segments in $\mathbb{R}_{o e^{\prime}}$ are infinitesimal relative to the nondegenerate segments in $\mathbb{R}_{o e}$, which in turn are infinitesimal relative to the nondegenerate segments in $\mathbb{R}_{o e^{\prime \prime}},{ }^{10}$ the ordered fields $\mathbb{R}_{o e}, \mathbb{R}_{o e^{\prime}}$, and $\mathbb{R}_{o e^{\prime \prime}}$ are all isomorphic to $\mathbb{R}$, and likewise for their corresponding Cartesian spaces $C_{3}\left(\mathbb{R}_{o e}\right), C_{3}\left(\mathbb{R}_{o e^{\prime}}\right)$, and $C_{3}\left(\mathbb{R}_{o e^{\prime \prime}}\right)$ with respect to $C_{3}(\mathbb{R})$.

While the above excursion is directed to non-Archimedean structures, it brings into focus the underappreciated fact that if $M$ and $M^{\prime}$ are models of $\mathcal{E}_{3}$, it is logically possible for the nondegenerate segments of $M$ to be infinitesimal relative to the nondegenerate segments of $M^{\prime}$. Accordingly, there is no logical difficulty in envisioning that the expansion $\left\langle\Pi, B, \equiv,\left(\left\langle\mathbb{R}_{x}^{3}, B_{x}, \equiv_{x}\right\rangle\right)_{x \in \mathbb{R}^{3}}\right\rangle$ of our first model of $\mathcal{E}_{3}$ models a hypothesized enriched Newtonian space in which the nondegenerate macrosegments - the nondegenerate segments modeled by the nondegenerate segments of $\langle\Pi, B, \equiv\rangle$-are finite relative to the standard meter, and the nondegererate microsegments - the nondegenerate segments modeled by the nondegenerate segments of the $\left\langle\mathbb{R}_{x}^{3}, B_{x}, \equiv_{\chi}\right\rangle$ 's-are infinitesimal relative to the standard meter. Indeed, whereas Hamlet figuratively proclaimed "I could be bounded in a nutshell and count myself a king of infinite space," a denizen of the hypothesized microspace modeled by $\left\langle\mathbb{R}_{x}^{3}, B_{x}, \equiv_{x}\right\rangle$ could literally make the same claim with "nutshell" replaced by "point of (enriched) Newtonian space"! of course, in this scenario the denizen of the microspace would be infinitesimal relative to the standard meter, despite the fact that the points of the microspace, together with the betweenness and equidistance relations defined thereon, model all of $\mathcal{E}_{3}$. In this setting there is plainly no reason to believe the spatial betweenness and equidistance relations defined on the macropoints - the points of the enriched Newtonian space - would be other than the familiar ones. Indeed, it is difficult to see why this would not be the case whether the nondegenerate segments of the $\left\langle\mathbb{R}_{x}^{3}, B_{x}, \equiv_{x}\right\rangle$ 's model segments that are finite, infinite, or infinitesimal relative to the standard meter; after all, in each such case the segments whose sole members are the $\left\langle\mathbb{R}_{\chi}^{3}, B_{\chi},{ }_{x}\right\rangle$ 's would be the degenerate segments of $\langle\Pi, B, \equiv\rangle{ }^{11}$

${ }^{10}$ Note that the distinct though isomorphic ordered fields $F_{o e}^{l}, F_{o e^{\prime}}^{l}$, and $F_{o e^{\prime \prime}}^{l}$ have a common underlying ordered additive group $G_{o}^{l}=\left\langle l,+_{l},<_{l}, 0 o\right\rangle$, and as such the ordered additive groups of $\mathbb{R}_{o e}, \mathbb{R}_{o e^{\prime}}$, and $\mathbb{R}_{o e^{\prime \prime}}$ are all subgroups of $G_{o}^{l}$, whose nonnull elements are comparable with respect to relative finitude and relative infinitude.

${ }^{11}$ In his aforementioned pioneering work on non-Archimedean geometry, Veronese $(1891,56, \S 9)$ introduced the notion of a nondegenerate segment that is indivisible relative to a system $S$ of nondegenerate segments. In essence, a nondegenerate segment $a b$ is indivisible relative to $S$ if $a b$ cannot be decomposed 
Besides the models of $\mathcal{E}_{3}$ with extended points constructed above, there is a wealth of other types of such models, including the aforementioned ones due to Huntington (see the Appendix). We will bring the main body of the paper to a close by drawing attention to a rather distinctive one, motivated by its inherent historical and mathematico-philosophical interest.

Since the time of Euclid it has been widely held that atomistic space and continuous space are incompatible (e.g., Hellman and Shapiro 2018, 190). After all, lines of atomistic space have been assumed to be discretely ordered-one space atom immediately following another-rather than densely ordered. However, if in our first construction of a model of $\mathcal{E}_{3}$ one replaces the copies of models of $\mathcal{E}_{3}$ indexed over $\mathbb{R}^{3}$ with copies of extended indivisible spatial atoms indexed over $\mathbb{R}^{3}$, one obtains a model of $\mathcal{E}_{3}$ whose points are extended indivisible spatial atoms. That is, one obtains a threedimensional Euclidean continuum of extended indivisible spatial atoms!

Acknowledgments. Earlier versions of this paper were presented at the Philosophy of Science Association Meeting, Chicago, Il., 2014, the 8th (University of Pittsburgh, Center for Philosophy of Science) International Fellows' Conference, University of Lund, Lund, Sweden, 2016, the 4th International Meeting of the Association for the Philosophy of Mathematical Practice, Universidad Federalda Bahia, Bahia/Salvador, Brazil, 2017, the 19th Midwest Philosophy of Mathematics Workshop, University of Notre Dame, 2018, and the 16th International Congress on Logic, Methodology and Philosophy of Science and Technology, Prague, Czech Republic, 2019. We are grateful to the various organizers for providing us those opportunities. We are also grateful to Patricia Blanchette, John Baldwin, and Geoffrey Hellman, whose collective questions, comments and suggestions substantially influenced the final version of the paper.

\section{References}

Baker, Catharine A., Norman D. Lane, Joseph W. Lorimer, and James A. Laxton. 1984. "Preordered Affine Hjelmslev Planes." Journal of Geometry 23 (1):14-44.

Benci, Vieri, Leon Horsten, and Sylvia Wenmackers. 2013. "Non-Archimedean Probability." Milan Journal of Mathematics 81 (2):121-51.

Benci, Vieri, Leon Horsten, and Sylvia Wenmackers. 2018. "Infinitesimal Probabilities.” British Journal for Philosophy of Science 69 (1):509-52.

Bernstein, Allen, and Frank Wattenberg. 1969. "Nonstandard Measure Theory." In Applications of Model Theory to Algebra, Analysis, and Probability, edited by W. A. J. Luxemburg, 171-85. New York: Holt, Rinehart and Winston.

Blumenthal, Otto. 1935. "Lebensgeschichte." In Gesammelte mathematische Abhandlungen 3, edited by David Hilbert, 388-429. Berlin: Springer.

Clifford, William. 1873. "Preliminary sketch of bi-quaternions." Proceedings of the London Mathematical Society 4 (November):381-95.

Du Bois-Reymond, Paul. 1882. Die allgemine Functionentheorie. Leipzig: Tübingen.

Ehrlich, Philip. 2014. “An Essay in Honor of Adolf Grünbaum's Ninetieth Birthday: A Reexamination of Zeno's Paradox of Extension." Philosophy of Science 81 (4):654-75.

Ehrlich, Philip. 2021. "Contemporary Infinitesimalist Theories of Continua and Their Late Nineteenthand Early Twentieth-Century Forerunners." In The History of Continua: Philosophical and Mathematical Perspectives, edited by Stewart Shapiro and Geoffrey Hellman, 502-70. Oxford: Oxford University Press.

into nondegenerate segments $a c$ and $c b$ in $S$. For example, in a non-Archimedean model of $\mathcal{P}_{3}$, each nondegenerate segment is indivisible relative to the class of segments that are infinite relative to it. One might also interpret this by saying that a nondegenerate segment in a non-Archimedean model of $\mathcal{P}_{3}$ is unextended relative to the class of segments that are infinite relative to it. There is an analogous notion of relative unextendedness that can be applied to the points in our models of $\mathcal{E}_{3}$ with extended points (as well as to hypothesized physical realizations thereof) that raises a number of interesting questions in the epistemology of geometry. We hope to address these at a later time. 
Enriques, Federigo. 1911. "Sui numeri non archimedei e su alcune loro interpretazioni." Bollettino della Mathesis, Società Italiana di Matematica 3 (a):87-105.

Gerla, Giangiacomo. 2021. "Point-Free Continuum." In The History of Continua: Philosophical and Mathematical Perspectives, edited by Stewart Shapiro and Geoffrey Hellman, 427-75. Oxford: Oxford University Press.

Gerla, Giangiacomo, and Rafał Gruszczyński. 2017. "Point-Free Geometry, Ovals, and Half-Planes.” Review of Symbolic Logic 10 (2):237-58.

Hartshorne, Robin. 2000. Geometry: Euclid and Beyond. New York: Springer-Verlag.

Hellman, Geoffrey, and Stewart Shapiro. 2018. Varieties of Continua: From Regions to Points and Back. Oxford: Oxford University Press.

Hilbert, David. 1899. Grundlagen der Geometrie. Leibzig: Teubner.

Hilbert, David. (1899) 1980. "Hilbert to Frege, December 29, 1899." In Gottleob Frege Philosophical and Mathematical Correspondence, edited by Gottfried Gabriel, Hans Hermes, Friedrich Kambartel, Christian Thiel, Albert Veraart, Brian Mcguinness, and Hans Kaal, 38-41. Oxford: Blackwell Publishers.

Hilbert, David. (1902) 1971. Foundations of Geometry. 10th ed. LaSalle, Illinois: Open Court.

Hjelmslev, Johannes. 1916. “Die Geometrie der Wirklichkeit.” Acta Mathematica 40 (1):3-66.

Hjelmslev, Johannes. 1923. "Die natürliche Geometrie." Abhandlungen aus dem Mathematischen Seminar der Universität Hamburg 2 (1):1-36.

Hjelmslev, Johannes. 1929. "Einleitung in die allgemeine Kongruenzlehre I,II." Det Kongelige Danske Videnskabernes Selskab Matematisk-Fysiske Meddelelser 8(11); 10(1).

Huntington, E. V. 1913. "A Set of Postulates for Abstract Geometry, Expressed in Terms of the Simple Relation of Inclusion." Mathematische Annalen 73 (4):522-59.

Klingenberg, Wilhelm. 1954. "Projektive und affine Ebenen mit Nachbarelementen." Mathematische Zeitschrift 60 (1):388-406.

Klingenberg, Wilhelm. 1954a. "Euklidische Ebenen mit Nachbarelementen." Mathematische Zeitschrift 61 (1):1-25.

Klingenberg, Wilhelm. 1955. "Desarguessche Ebenen mit Nachbarelementen." Abhandlungen aus dem Mathematischen Seminar der Universität Hamburg 20 (1-2):97-111.

Kock, Anders. 1981. Synthetic Differential Geometry. 2nd Ed. Cambridge: Cambridge University Press.

Kreuzer, Alexander. 1987. "Hjelmslev-Räume." Results in Mathematics 12 (1-2):148-56.

Kreuzer, Alexander. 1988. Projective Hjelmslev-Räume. PhD diss., Technische Universität, München.

Levi-Civita, Tullio. 1892-93. "Sugli infiniti ed infinitesimi attuali quali elementi analitici." Atti del R. Istituto Veneto di Scienze Lettre ed Arti, Venezia (Serie 7) 4 (Sem. 1º):1765-1815.

Levi-Civita, Tullio. 1898. "Sui numeri transfiniti." Atti della Reale Accademia dei Lincei, Classe di scienze fisiche, matematiche e naturali, Rendiconti, Roma (Serie Va) 7 (Sem. 1º):91-96, 113-21.

Lingenberg, R., and A. Bauer. 1974. "Affine and Projective Planes." In Fundamentals of Mathematics, Volume II: Geometry, edited by H. Benhnke, F. Bachmann, K. Fladt, and H. Kunle, 64-111. Cambridge, MA: MIT Press.

Menger, Karl. 1940. “Topology without Points.” Rice Institute Pamphlet 27:80-107.

Monk, J. Donald. 1976. Mathematical Logic. New York: Springer-Verlag.

Robinson, Abraham. 1973. "Function Theory on Some Nonarchimedean Fields." American Mathematical Monthly 80 (no. 6, part I):8-109.

Schwabhäuser, Wolfram, Wanda Szmielew, and Alfred Tarski. 1983. Metamathematische Methoden in der Geometrie. Berlin: Springer-Verlag.

Tarski, Alfred. (1929) 1983. "Foundations of the Geometry of Solids." In Logic, Semantics, Metamathematics. Papers from 1923 to 1938. Translated by J. H. Woodger. Second edition edited and introduced by John Corcoran, 24-29. Indianapolis, IN: Hackett Publishing Co.

Tarski, Alfred. 1959. "What is Elementary Geometry?" In The Axiomatic Method, with Special Reference to Geometry and Physics, edited by Leon Henkin, Patrick Suppes, and Alfred Tarski, 16-29. Amsterdam: North-Holland Publishing Company.

Torretti, Roberto. 1984. Philosophy of Geometry from Riemann to Poincaré. Dordrecht: D. Reidel Publishing Company.

Varzi, Achille C. 2021. "Points As Higher-Order constructs: Whitehead's Method of Extensive Abstraction." In The History of Continua: Philosophical and Mathematical Perspectives, edited by Stewart Shapiro and Geoffrey Hellman, 347-74. Oxford: Oxford University Press. 
Veblen, Oswald. (1911) 1955. "The Foundations of Geometry." In Monographs on Topics of Modern Mathematics, edited by J. W. A. Young, 3-51. Longmans, Green and Company. Reprint, New York: Dover Publications, Inc.

Veronese, Giuseppe. 1891. Fondamenti di geometria a più dimensioni e a più specie di unità rettilinee esposti in forma elementare. Padova: Tipografia del Seminario.

\section{Appendix: Huntington's anticipation}

As was noted in the Introduction, to establish the consistency of his postulates for ordinary threedimensional Euclidean geometry, Huntington introduces a "geometry of points of finite size" (1913, 524). Huntington's formulation is based on two primitives: the notion of a sphere-spheres being the members of a class $K$-and a (transitive, irreflexive) binary relation $R$ of inclusion (1913, 537). Points are defined as spheres which do not include any other sphere $(1913,529)$.

Following his definition of point, Huntington goes on to add: "It may be noticed that there is nothing in this definition, or in any of our work, which requires our 'points' to be small; for example, a perfectly good geometry is presented by the class of all ordinary spheres whose diameters are not less than one inch; the 'points' of this system are simply the inch-spheres" (1913, 529-530).

After developing the remainder of his theory, Huntington constructs a class of models for his postulates making use of the just noted idea as follows:

[L]et $S(a, b, c, r)$ denote the class of all triads of real numbers $x, y, z$, which satisfy the equation

$$
(x-a)^{2}+(y-b)^{2}+(z-c)^{2} \leq r^{2},
$$

where $a, b, c, r$ are real numbers, and $r$ is not less than a certain fixed number $g$ (positive or zero).

We take as our class $K$ the totality of all such $S$ 's, and we define the relation $R$ between any two of these $S$ 's by agreeing that

$$
S\left(a^{\prime}, b^{\prime}, c^{\prime}, r^{\prime}\right) R S\left(a^{\prime \prime}, b^{\prime \prime}, c^{\prime \prime}, r^{\prime \prime}\right)
$$

when and only when $r \prime \neq r^{\prime \prime}$ and every triad $x, y, z$ which satisfies the relation

$$
\left(x-a^{\prime}\right)^{2}+\left(y-b^{\prime}\right)^{2}+(z-c)^{2} \leq r^{\prime} 2
$$

satisfies also the relation

$$
\left(x-a^{\prime \prime}\right)^{2}+\left(y-b^{\prime \prime}\right)^{2}+\left(z-c^{\prime \prime}\right)^{2} \leq r^{\prime \prime} 2 .
$$

In this system $(K, R)$, the 'points' are the elements of the form $S(a, b, c, g)$; and it is not hard to show that all the postulates are satisfied.

Moreover, as he goes on to add: "In the language of analytic geometry, this system is simply the system of spheres whose radii are not less than $g$, where, in the most familiar case, $g=0$. It is interesting to observe that any other value of $g$ is equally legitimate, so that we may speak of a perfectly rigorous geometry in which the 'points', like the school-master's chalk-marks on the blackboard, are of definite, finite size, and the 'lines' and 'planes' of definite finite thickness" (1913, 540).

Huntington's system is the first in a long line of axiomatizations of ordinary three-dimensional Euclidean geometry in which the concept of a point is treated as a defined term, as opposed to a primitive (e.g., Hellman and Shapiro 2018; Gerla 2021); and with the sole exception of Torretti (1984, 243-46), from which we recently learned of Huntington's extended points, all of the references to it we had been aware of, including Tarski ([1929] 1983, 26), Menger (1940, 84) Gerla and Gruszczyński (2017), and Varzi (2021, $348)$, refer to it primarily for this reason. Unfortunately, as Torretti $(1984,244)$ aptly notes, Huntington's system, especially his half page (!) definition of congruence, is "unpleasantly complicated," and we suspect it is for this reason that the details of Huntington's system, including his "points of finite size," have been largely forgotten.

Readers seeking an overview of Huntington's system may consult Torretti (1984, 243-46). Given the limitation of space we will merely introduce Huntington's notions of line segment and line, show how a betweenness relation may be defined thereon, and specify the relations that exist between the betweenness and congruence relations in Huntington's system and those in $C_{3}(\mathbb{R})=\left\langle\mathbb{R}^{3}, B_{\mathbb{R}}, \equiv \mathbb{R}\right\rangle$. 
For any two points $a$ and $b$ as defined above, Huntington defines the line segment $[a b]$ (or [ba]) as the set of all points $x$ such that every sphere that contains points $a$ and $b$ also contains $x$, and he further defines the corresponding line $a b$ as the union of $[a b]$ and its two prolongations: $\{x: x$ is a point and $a \in[x b]\}$ and $\{x: x$ is a point and $b \in[a x]\}(1913,530-531)$. In the Cartesian space $C_{3}(\mathbb{R})$, a line so defined having points with fixed radius $r>0$ consists of the set of all spheres of radius $r$ whose centers lie on a standard line in $C_{3}(\mathbb{R})$, and, for the case where $r=0$, it coincides with a standard line itself. ${ }^{12}$ Accordingly, if we let $\equiv *$ denote Huntington's congruence relation on segments and define a nonstrict betweeness relation $B^{*}$ (à la Tarski) on triples of points $a, b, c$ by the condition: $B_{a b c}^{*}$ if and only if $b \in[a c]$, then the relation between these constructs and their Cartesian counterparts is given by the following conditions for all points $a, b, c, d$ :

$$
\begin{gathered}
B_{a b c}^{*} \text { if and only if } B_{\mathbb{R}} a^{\prime} b^{\prime} c^{\prime}, \\
a b \equiv{ }^{*} c d \text { if and only if } a^{\prime} b^{\prime} \equiv{ }_{\mathbb{R}} c^{\prime} d^{\prime},
\end{gathered}
$$

where $a^{\prime}, b^{\prime}, c^{\prime}, d^{\prime}$ are the centers of the points (i.e., spheres) $a, b, c, d$ when $r>0$ and coincide with $a, b, c, d$ when $r=0$.

As our earlier remarks would suggest, and as Huntington makes clear, all of the points in his models of three-dimensional Euclidean geometry, even those with extended points, are assigned null lengths, and all nondegenerate segments are assigned positive real lengths (Huntington 1913, 535, 546-47). The standard points inside Huntington's extended spherical points and the betweenness and congruence relations defined thereon are, of course, not part of Huntington's models. However, using many-sorted expansions of his models (analogous to those employed in section 9), they can be brought to light.

\footnotetext{
${ }^{12}$ If we assume a standard point is a sphere that coincides with its center, then for $r \geq 0$ this follows from the simple fact that a point $X$ is not on the segment joining the centers of spheres $S^{\prime}$ and $S^{\prime \prime}$ of radius $r$ if and only if there is a sphere $S$ of radius $r$ with center $X$ and a sphere $S^{*}$ containing $S^{\prime}$ and $S^{\prime \prime}$ but not $S$ (Huntington 1913, 530, Fig. 1).
}

Cite this article: Ehrlich, Philip. 2022. “Are Points (Necessarily) Unextended?" Philosophy of Science 89 (4): 784-801. https://doi.org/10.1017/psa.2021.47 